\title{
Assessment of nitrogen fluence from the divertor plasma in nitrogen seeded discharges
}

Thomas Reichbauer ${ }^{\mathrm{a}}$, Aleksander Drenik ${ }^{\mathrm{a}}$, Rachael McDermott ${ }^{\mathrm{a}}$, Volker Rohde ${ }^{\mathrm{a}}$ and the ASDEX Upgrade Team

aMax-Planck-Institut für Plasmaphysik, 85748 Garching b. München, Germany

\begin{abstract}
Impurity seeding will be mandatory for ITER to protect the divertor tiles from excessive heat loads. Nitrogen seems to be a possible candidate due to its good radiation properties. The $\mathrm{N}$ content in the divertor plasma is crucial for cooling by radiation but is difficult to characterize.
\end{abstract}

To assess the nitrogen fluence from the divertor plasma in nitrogen seeded discharges of ASDEX Upgrade (AUG), the concentration of two nitrogen containing molecules, ammonia and molecular nitrogen $\left(\mathrm{N}_{2}\right)$ was measured. The $\mathrm{N}_{2}$ measurements were strongly affected by local effects which indicates that a significant fraction of the puffed $\mathrm{N}_{2}$ did not enter the plasma. In contrast, the measured ammonia fluence is proportional to the integrated nitrogen core density. This leads to the possibility of gaining the nitrogen fluence of the plasma, with the help of the created ammonia, for nitrogen seeded discharges.

In discharges where the wall inventory was the only source of nitrogen in the plasma, $50 \%$ of the nitrogen was converted to ammonia, in the inner divertor. This allowed for the assessment of the nitrogen atom fluence from the plasma based on the detected ammonia in $\mathrm{N}_{2}$-seeded discharges.

\section{Introduction}

In fusion devices, a certain concentration of low- $Z$ impurities in the plasma edge is required to mitigate the divertor heat loads of high power $\mathrm{H}$-mode discharges by dissipating the plasma-stored energy through radiation [1]. In devices with fully metallic first walls, like ASDEX Upgrade and JET, the intrinsic impurity level is not sufficient, and impurity seeding is required to protect the tiles from overloads and damages. Impurity seeding will thus also be required in the upcoming fusion device ITER. In ASDEX Upgrade nitrogen gives the best results in terms of radiation distribution in the plasma edge and also improvement of the confinement [14]. Therefore, nitrogen is also considered as a seeding gas in plasma scenarios at ITER.

Previous research results showed that up to $8 \%$ of the seeded nitrogen was converted to ammonia [2]. It was also shown that the nitrogen concentration in the 
plasma was strongly affected by the wall inventory [11] and therefore could vary significantly at the same $\mathrm{N}_{2}$-seeding rates [7]. While the nitrogen concentration in the core is measured with charge exchange recombination spectroscopy (CXRS) [9], the nitrogen concentration in the divertor plasma, which is crucial to the radiation efficiency, remains uncharacterised.

\section{Experimental setup}

Residual gas analysis at ASDEX Upgrade is performed with commercially available devices, however certain adaptations are necessary to the tokamak environment: magnetic fields of $2.5 \mathrm{~T}$ to confine the plasma will disturb the quadrupole filters of the mass spectrometers, and the divertor pressures reaching up to $1 \mathrm{~Pa}$ [7] will exceed their operational pressure range. Therefore, all analysers are differentially pumped and built into an iron box which acts as magnetic shielding. The pressure at the outer divertor pressure gauges is $10^{-4} \mathrm{~Pa}$ between and about $1 \mathrm{~Pa}$ during discharges. Figure 1 illustrates the positions of the used mass spectrometers and pressure gauges for the data analysis and the pumping system.

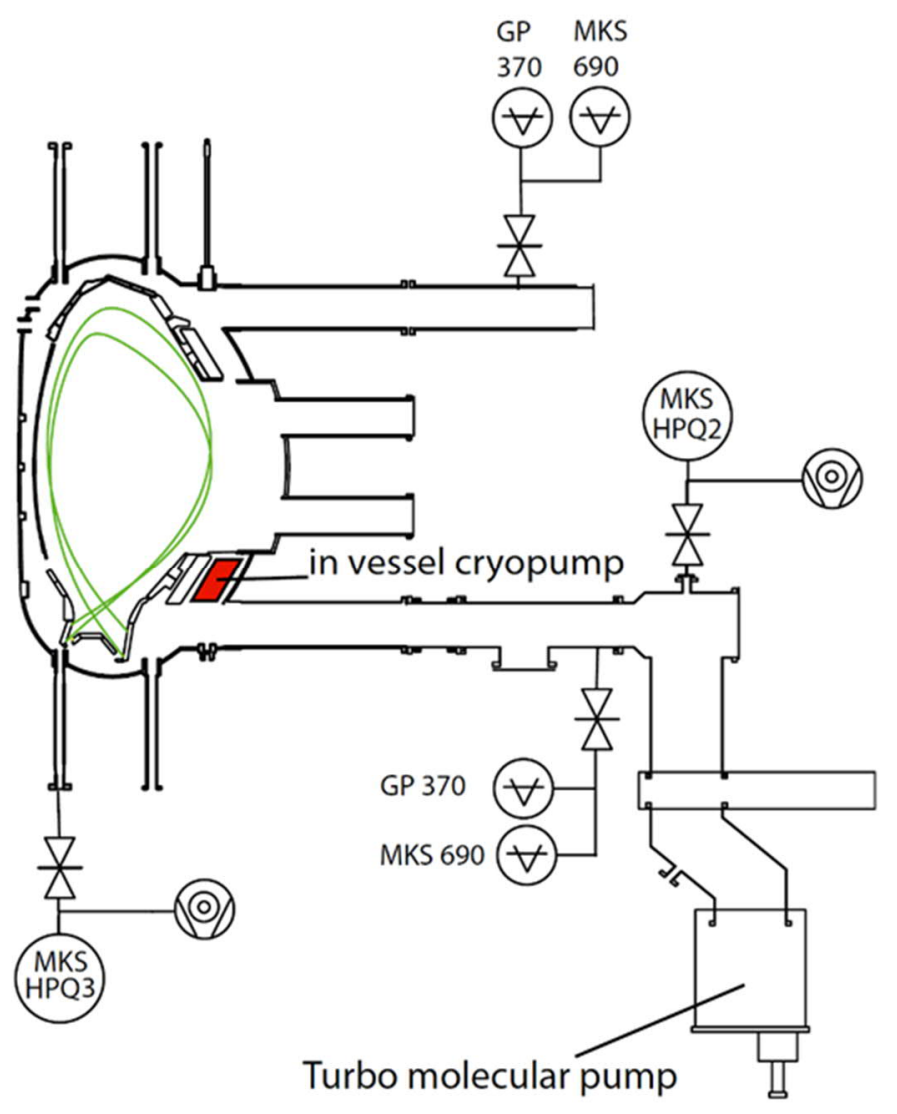

Figure 1. Cross section of AUG with pumps, pressure gauges (capacitance manometers MKS 690, hot cathode ionization gauges GP 370) and mass spectrometers

Data from two mass spectrometers (MS) are used in this study. One of them is looking at the lower inner divertor and the other one in the lower outer divertor. Both devices are produced by MKS (HPQ2 and HPQ3) $[15,16]$ and are equipped with a short quadrupole filter which allows for a relatively high pressure limit of $0.1 \mathrm{~Pa}$ and reduces the sensitivity to external magnetic fields. Next to the outer divertor MS, a 
capacitance manometer (MKS 690) and a hot cathode ionization manometer (Granville Phillips 370) are installed for uninterrupted measurements of the neutral gas pressure both during the discharge phase when it can reach up to $1 \mathrm{~Pa}$ in the divertor, as well as between the discharges when it is of the order of $10^{-4} \mathrm{~Pa}$. The same group of gauges is also installed in the midplane region which was used for calibration.

During discharges the mass spectrometers were set to measure intensities at discrete mass-to-charge ratios with a time resolution of about $0.5 \mathrm{~s}$ per scan. Between discharges they were set to acquire spectra with a resolution of $0.06 \mathrm{AMU}$, and an increased sensitivity. The acquisition time was around $30 \mathrm{~s}$ per spectrum. A detailed description of the measurement process can be found in [2].

\section{Analysis of RGA recordings}

Analysis of the impurities in neutral gas has already been described in detail for AUG and JET $[5,12]$. In this analysis, the main focus was on nitrogen containing molecular species, i.e. molecular nitrogen $\left(\mathrm{N}_{2}\right)$ and ammonia. While the detection of $\mathrm{N}_{2}$ in the neutral gas is straight forward as it is the only molecule with a $28 \mathrm{AMU} / \mathrm{e}_{0}$ mass-tocharge ratio, more attention has to be paid to the detection of ammonia.

In ASDEX Upgrade deuterium (D) is used as plasma fuel, however non-zero quantities of protium $(\mathrm{H})$ are always present. In a mixed $\mathrm{H}-\mathrm{D}$ environment, the mass spectra of ammonia overlap with those of water and methane, which are always present in the neutral gas during discharges [2, 13]. The contributions of each of the species is deconvoluted from the RGA recordings using the model of Price and Iglesia [4], which assumes an average hydrogen isotope ratio for each molecule. The isotope ratio then defines the probability distribution of the molecule's isotopologues, resulting in an overall cracking pattern, described by:

$$
C P=\mathcal{A} \cdot \vec{f},
$$

where $\vec{f}$ is the cracking pattern of a purely hydrogenated molecule and $\mathcal{A}$ is the probability matrix. A detailed description of the deconvolution can be found in [5].

\subsection{Calibration of mass spectrometers}

In order to quantify the impurities, detected by the RGAs, the sensitivity of the instruments has to be determined in-situ. To improve the reliability of detection of ammonia, the cracking patterns of ammonia, as well as of water and methane, have to be measured for each instrument, as well.

Key parameters for the interpretation of RGA data are the calibration factor and the cracking pattern. To quantify the impurity gases during discharges calibration factors are needed which connect the measured intensities to the chamber partial pressures of each gas. On the other hand, cracking patterns for ammonia, methane and water are needed for deconvolution of these gases. Such parameters must be obtained for each mass spectrometer individually. 


\subsubsection{Standard calibration}

To measure the instrument sensitivity and cracking patterns, nitrogen and methane were puffed into the plasma vessel while all valves to the turbo molecular pumps were shut and the cryo panels were warmed up. This resulted in a stable and homogeneous pressure distribution which allowed a straight-forward relation between the measured intensity and the pressure in the main chamber (measured with an absolute pressure gauge). The gas injections were performed stepwise, where each injection step resulted in an increase of the vessel pressure of approximately $0.5 \cdot 10^{-3}$ $\mathrm{Pa}$, up to the maximum pressure of $10^{-2} \mathrm{~Pa}$, as shown in Figure 2. From each of these injection steps, the average values of the vessel pressure and the recorded intensity were used to determine the calibration factor. The mass spectrometers were acquiring data in the profile mode, i.e. they were recording continuous spectra in the $1-50 \mathrm{AMU} / \mathrm{e}_{0}$ range. The intensities at each $\mathrm{m} / \mathrm{z}$ value, shown in Figure 2, represent the maximum of the spectral peak at that particular $\mathrm{m} / \mathrm{z}$ value. Figure 2 shows the intensities at expected peaks of the $\mathrm{CH}_{4}$ molecule (12 to $16 \mathrm{AMU} / \mathrm{e} 0$ ) as well as those related to impurities $\left(\mathrm{H}_{2} \mathrm{O}\right.$ at $17 \mathrm{AMU} / \mathrm{e}_{0}, \mathrm{~N}_{2}$ at $28 \mathrm{AMU} / \mathrm{e}_{0}$ and $\mathrm{O}_{2}$ at 32 $\mathrm{AMU} / \mathrm{e}_{0}$ ). Among those, $\mathrm{H}_{2} \mathrm{O}$ showed the same behaviour as the $\mathrm{CH}_{4}$ signals whereas $\mathrm{N}_{2}$ showed less correlation to the total pressure and only negligible content of $\mathrm{O}_{2}$ was measured.

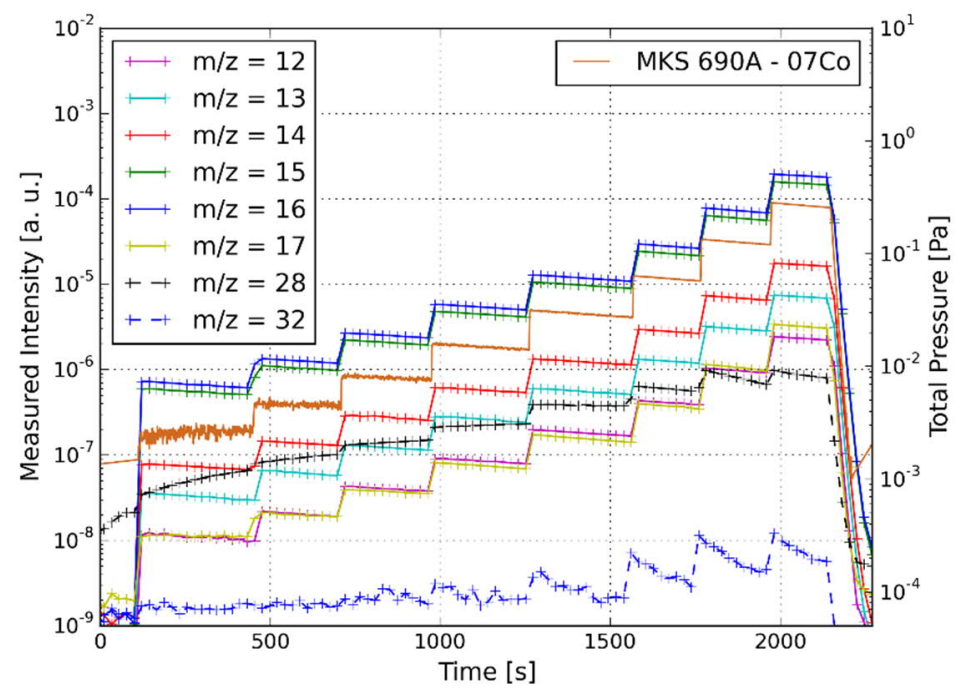

Figure 2. Calibration measurement of the inner divertor mass spectrometer, done for isotopically pure methane $\left(\mathrm{CH}_{4}\right)$ in the vacuum vessel of AUG with the corresponding pressures measured by MKS 690 in the midplane.

It relates the measured intensity (arb. u.) to the partial pressure of the gas in the main chamber $(\mathrm{Pa})$.

Due to their high sticking probabilities [2] and the related technical difficulties, the calibration procedures were not performed with ammonia and water as they were not expected to provide the same stable pressure behaviour as the non-sticking gasses. Instead, the calibration for water was performed with the residual atmosphere (i.e. at base pressure), in which water was the main component. While a direct calibration for ammonia was not possible, its cracking pattern was nevertheless determined, as described in the following sub-section. 
The measured cracking patterns for hydrogenated methane, ammonia and water can be seen in Table 2 and 3 for the inner and outer divertor devices. The tables list the cracking patterns of fully hydrogenated molecules, in the appropriate mass ranges (12 - $16 \mathrm{AMU} / \mathrm{e}_{0}$ for $\mathrm{CH}_{4}, 14-17 \mathrm{AMU} / \mathrm{e}_{0}$ for $\mathrm{NH}_{3}$ and $16-18 \mathrm{AMU} / \mathrm{e}_{0}$ for $\mathrm{H}_{2} \mathrm{O}$ ).

\begin{tabular}{|c|c|c|c|}
\hline $\mathrm{m} / \mathrm{z}$ & $\mathrm{CH}_{4}$ & $\mathrm{NH}_{3}$ & $\mathrm{H}_{2} \mathrm{O}$ \\
\hline 12 & 0.016 & - & - \\
\hline 13 & 0.050 & - & - \\
\hline 14 & 0.109 & 0 & - \\
\hline 15 & 0.826 & 0.034 & - \\
\hline 16 & 1 & 0.630 & 0.100 \\
\hline 17 & - & 1 & 0.300 \\
\hline 18 & - & - & 1 \\
\hline
\end{tabular}

Table 2. Calibrated cracking patterns of methane, ammonia and water for inner divertor device HPQ3 (MKS114-M0314025)

\begin{tabular}{|c|c|c|c|}
\hline $\mathrm{m} / \mathrm{z}$ & $\mathrm{CH}_{4}$ & $\mathrm{NH}_{3}$ & $\mathrm{H}_{2} \mathrm{O}$ \\
\hline 12 & 0.022 & - & - \\
\hline 13 & 0.071 & - & - \\
\hline 14 & 0.152 & 0 & - \\
\hline 15 & 0.855 & 0.039 & - \\
\hline 16 & 1 & 0.790 & 0.070 \\
\hline 17 & - & 1 & 0.260 \\
\hline 18 & - & - & 1 \\
\hline
\end{tabular}

Table 3. Calibrated cracking patterns of methane, ammonia and water for outer divertor device HPQ2 (LM75-00308013)

\subsubsection{Calibration for ammonia}

Owing to its high sticking probability, the outgassing of discharge-produced ammonia is significantly longer than of water and methane. After the end of the divertor phase which drives the high divertor pressure, the pressures of impurities (water, methane and nitrogen) drop to background levels in about 2 seconds, while the pressure of ammonia decays with an order of magnitude longer characteristic time [5, 7]. Thus, after discharges with high $\mathrm{N}_{2}$-seeding rates, the pressures of ammonia in the outgassing phase were high enough to provide adequate data for the determination of ammonia's cracking pattern. 


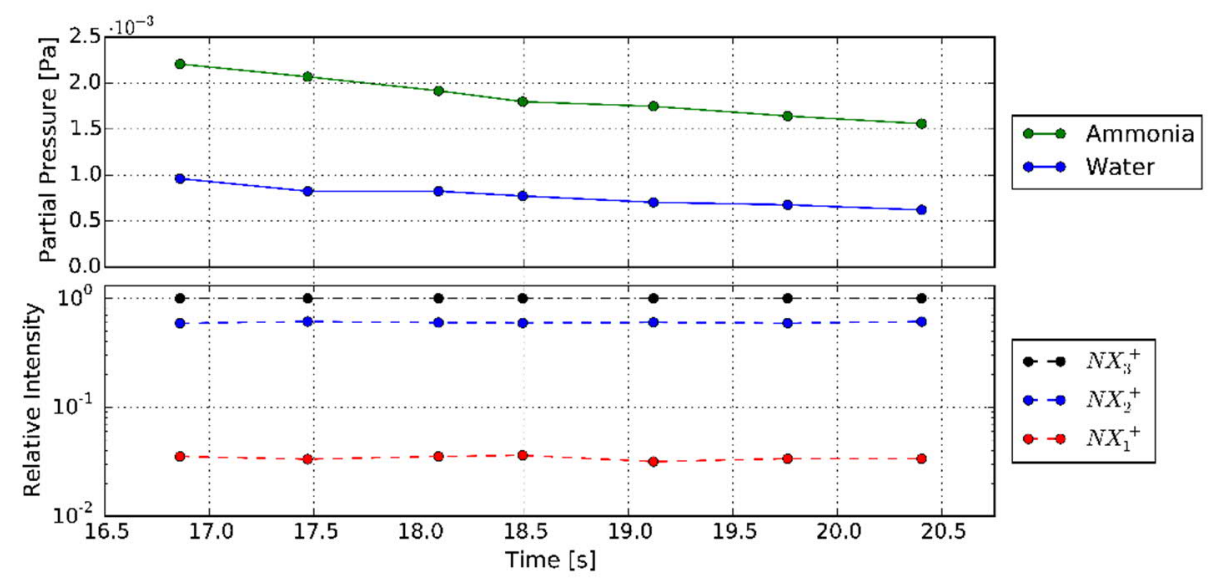

Figure 3. Fitted partial pressures and cracking pattern to the last 7 measurement points during Peak Jump mode for discharge 34267 and the inner divertor mass spectrometer. The time is measured from the start of the discharge which ended at $8 \mathrm{~s}$. The cracking pattern is indicated by the relative intensity of the detected ionic species $\left(\mathrm{NX}_{3}{ }^{+}, \mathrm{NX}_{2}{ }^{+}, \mathrm{NH}_{1}{ }^{+}\right)$, where $\mathrm{X}$ can be either a $\mathrm{H}$ or a $\mathrm{D}$ atom.

To determine the cracking pattern of ammonia, the deconvolution routine was used to calculate the cracking pattern of ammonia. 6 unknown variables were fitted: the partial pressures and $\mathrm{HD}$ ratios of water and ammonia and 2 cracking pattern entries of ammonia. This routine was applied to the last 7 measurement steps of highly seeded discharge \#34267. In Figure 3 the fitted partial pressures and the cracking pattern for the last 7 measurements of Peak Jump mode can be seen for the inner divertor. As supposed at the beginning, ammonia is the most abundant impurity next to water. The routine returned a stable cracking pattern over all data points. The calibrated cracking pattern of ammonia can be seen in Table 2 and 3 for the inner and outer divertor device. It is noteworthy that although the devices have the same type of ionisation cell, filter and detector, there is a considerable difference in the measured cracking patterns, which highlights the need of calibration for every device used in such studies.

\section{Assessment of nitrogen fluence from the divertor plasma with residual gas analysis}

With the assumption that the gas mixture that reaches the RGA acts like an ideal gas, the measured intensity (in arb. u.) can be connected to the particle flux density, $F$, (in $\mathrm{m}^{-2} \mathrm{~s}^{-1}$ ) by:

$$
\mathrm{F}=\frac{1}{4} v_{t h} n=\frac{1}{4} v_{t h} \frac{p}{k_{B} T}=\frac{1}{4} v_{t h} \frac{C \cdot I}{k_{B} T},
$$

where $v_{t h}$ is the thermal velocity, $k_{B}$ the Boltzmann constant, $T$ the gas temperature which is assumed to be $300 \mathrm{~K}, C$ is the calibration factor which connects the measured intensity of the RGA to the measured partial pressure of MKS 690 . Previous studies have shown that the transport of ammonia from the divertor to the RGAs is significantly slowed down because of the sticking of ammonia to the pipework surfaces [7] and that the rise and decay time of the partial pressure are of the order of seconds. To obtain the information of the total fluence, $\Phi$, the particle flux densities are integrated over the duration of the discharge and the subsequent outgassing phase: 


$$
\Phi=\int F(t) d t
$$

By integration of the flux density the particle fluence from the divertor plasma of each gas can be calculated and compared with other measurements. The fluence of nitrogen atoms is then a sum of the fluence of nitrogen atoms in molecular nitrogen $\left(\mathrm{N}_{2}\right)$ and in ammonia $\left(\mathrm{NH}_{3}\right)$ :

$$
\Phi_{\mathrm{N}}=2 \cdot \Phi_{\mathrm{N}_{2}}+\Phi_{\mathrm{NH}_{3}}
$$

\subsection{Molecular nitrogen}

Even in non-seeded discharges, $\mathrm{N}_{2}$ is always present in the neutral gas in detectably high concentrations. With $\mathrm{N}_{2}$ seeding, the pressure of $\mathrm{N}_{2}$ in the neutral gas measured with divertor RGAs increases by at least an order of magnitude [2, 13]. At JET, the amount of detected $\mathrm{N}_{2}$ has been shown to be generally proportional to the amount of injected $\mathrm{N}_{2}$ [13].

At $A \cup G, N_{2}$ is injected into the divertor area through a system of four toroidally distributed pairs of inlet valves. The locations of the valves, together with the location of the divertor RGAs are shown in Figure 4. The number of valves used to inject $\mathrm{N}_{2}$

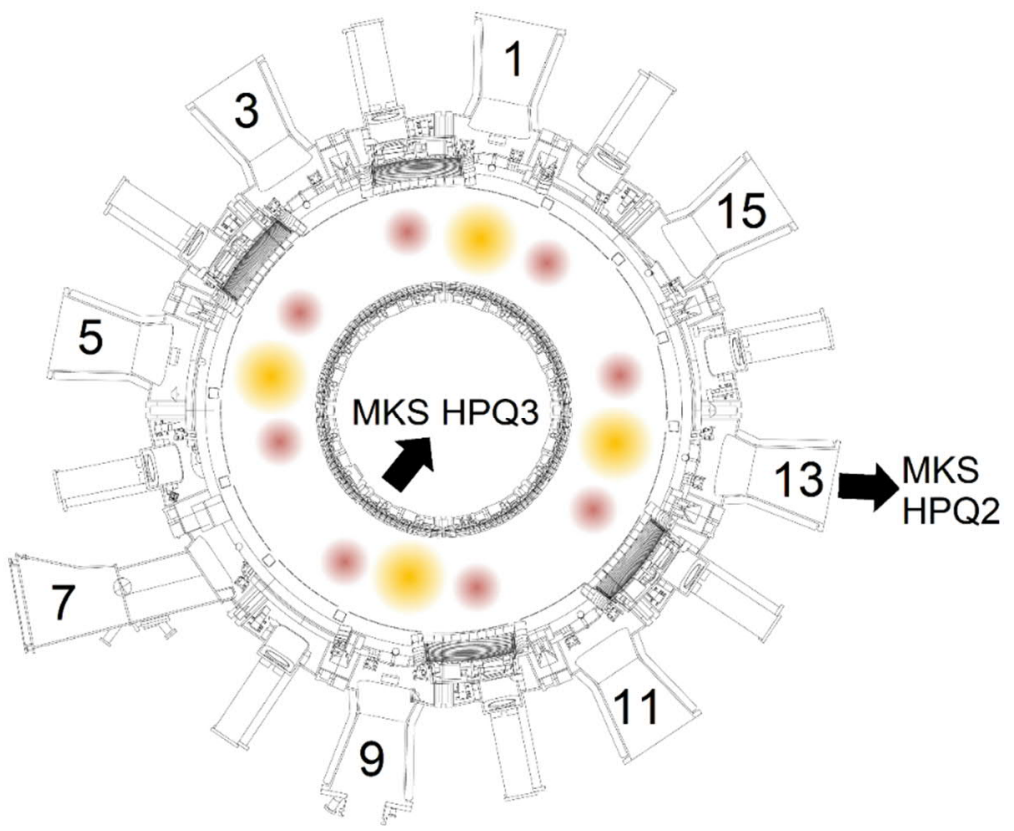

Figure 4. Positions of seeding valves of $\mathrm{N}_{2}$ (red) and $\mathrm{D}_{2}$ (yellow) and RGAs in ASDEX Upgrade

depends on the discharge setup. The response of the inner RGA to the amount of seeded $\mathrm{N}_{2}$ is shown in Figure 5. One of the $\mathrm{N}_{2}$ injection valves is located in direct vicinity of the diagnostics port through which the inner divertor is connected to the $A U G$ vessel. As shown in Figure 5, the response of the RGA is significantly larger when the valve is used than when it is not. A similar response to the valve location 


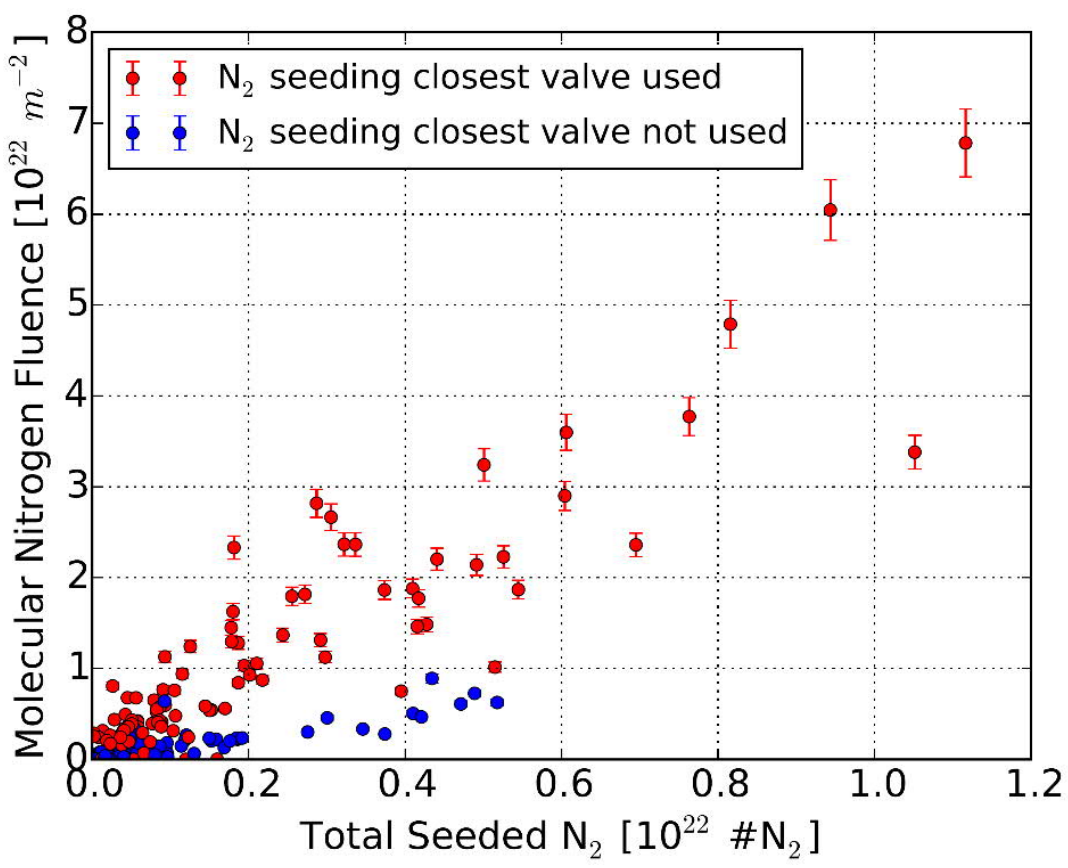

Figure 5. Fluence of nitrogen measured with the inner divertor RGA, plotted against amount of seeded $\mathrm{N}_{2}$

was observed with the outer divertor RGA, in relation to the valve closest to its diagnostic port [10]. The most likely explanation for this discrepancy is that a significant fraction of the puffed $\mathrm{N}_{2}$ bypasses the plasma and goes straight to the pumping lines of the RGAs.

The discrepancy between the concentration of $\mathrm{N}_{2}$ in the neutral gas and nitrogen concentration in the plasma is further outlined in the relation between the $\mathrm{N}_{2}$ detected by the RGA and the nitrogen concentration in the core, measured by charge exchange recombination spectroscopy (CXRS) [9], plotted in Figure 6. In this case, the types of discharges considered were $\mathrm{N}_{2}$-seeded discharges (using all seeding valves) and the so called legacy discharges. A legacy discharge is a non-seeded discharge performed after $\mathrm{N}_{2}$-seeded ones, i.e. with a non-negligible nitrogen inventory in the machine walls. In such discharges, the nitrogen removed from the walls is the only source of the nitrogen in the plasma and the RGA signals are not distorted by the bypass of the puffed $\mathrm{N}_{2}$. As shown in Figure 6, the detected $\mathrm{N}_{2}$ in the neutral gas is larger by an order of magnitude at the same core $\mathrm{N}$ densities in seeded discharges. While the detected amounts of $\mathrm{N}_{2}$ in the neutral gas have been shown to be subject to evolution of the nitrogen wall inventory and the resulting changes in the plasma parameters [7], this alone does not sufficiently explain the large difference in the trends shown in Figure 6 . The discrepancy in the relation between the $\mathrm{N}_{2}$ in the neutral gas and $\mathrm{N}$ in the plasma in seeded and legacy discharges is thus a strong indication of a bypass of the seeded $\mathrm{N}_{2}$ which is pumped into the RGA connection ports without interacting with the plasma beforehand. 


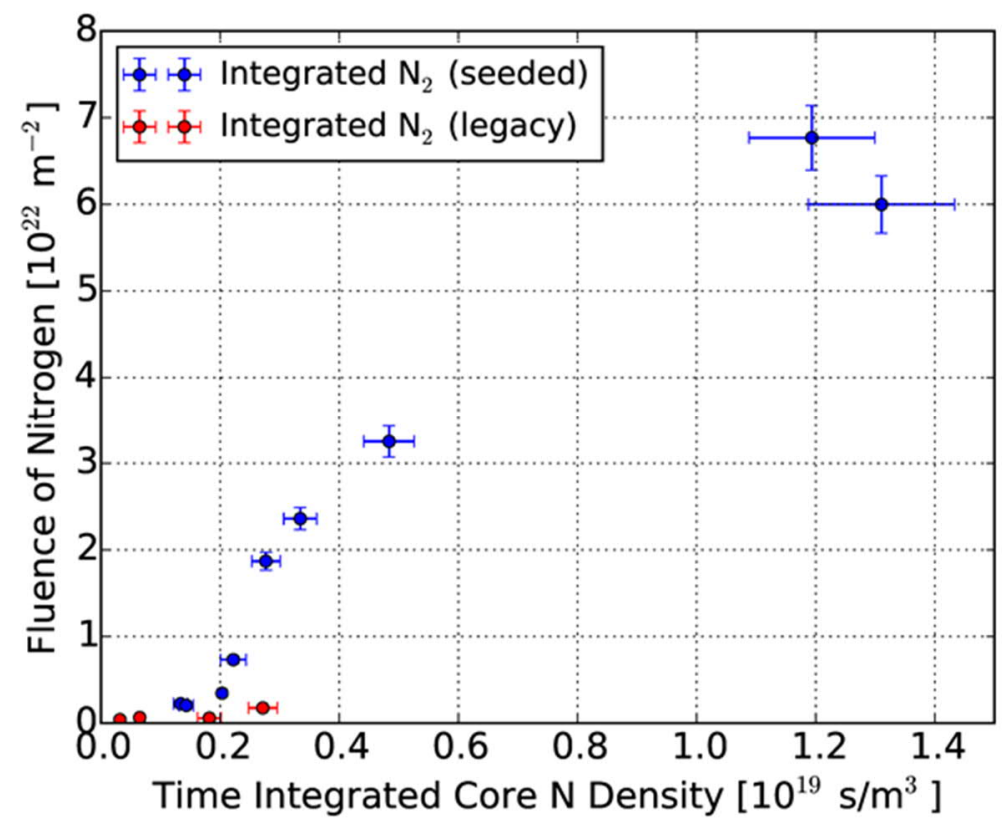

Figure 6. Fluence of $\mathrm{N}_{2}$ measured with the inner divertor RGA, plotted against pulse-integrated core $\mathrm{N}$ density, for seeded and legacy discharges

\subsection{Ammonia}

The fluence of ammonia, measured with the inner divertor RGA, is plotted as a function of the amount of seeded $\mathrm{N}_{2}$ in Figure 7 , and as a function of the pulseintegrated core $\mathrm{N}$ density in Figure 8 . Unlike molecular $\mathrm{N}_{2}$, the behaviour of ammonia shows no local effects, nor is there any notable difference between seeded and legacy discharges.

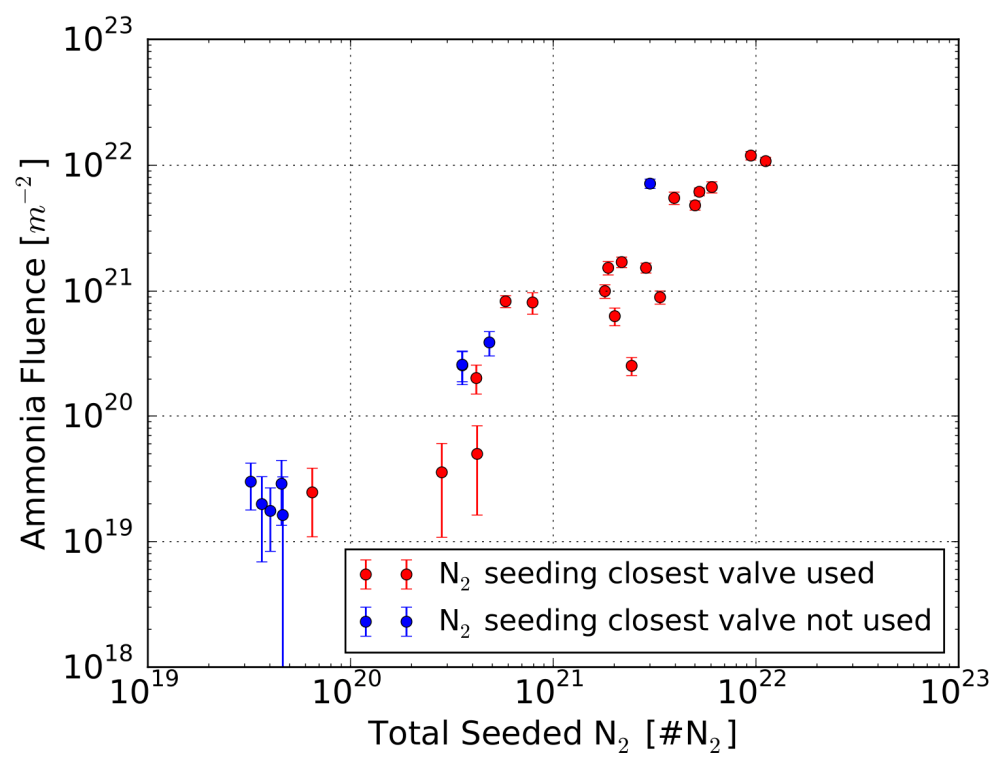

Figure 7. Fluence of ammonia measured with the inner divertor RGA, plotted against total injected number of $\mathrm{N}_{2}$ 
For the same discharges as in Figure 5, the fitting routine was used to calculate the fluence of ammonia. The comparison between the measured fluence of ammonia and the total seeded nitrogen (Figure 7) shows no discrepancy in the usage of different seeding valves. Ammonia seems to be spread toroidal symmetrical and therefore no local seeding effect occurs, in contrast to measured nitrogen.

Comparing the ammonia fluence of the RGAs of seeded and legacy discharges with the integrated nitrogen content in the core plasma by CXRS shows a linear behaviour (see Figure 8). The ammonia measurement is correlated to the nitrogen density in the core plasma, it is independent on local and bypass effects as seen for the measured nitrogen fluence.

Therefore, in contrast to nitrogen, ammonia in the neutral gas can be a good indicator for the fluence of nitrogen from the divertor plasma to the RGAs.

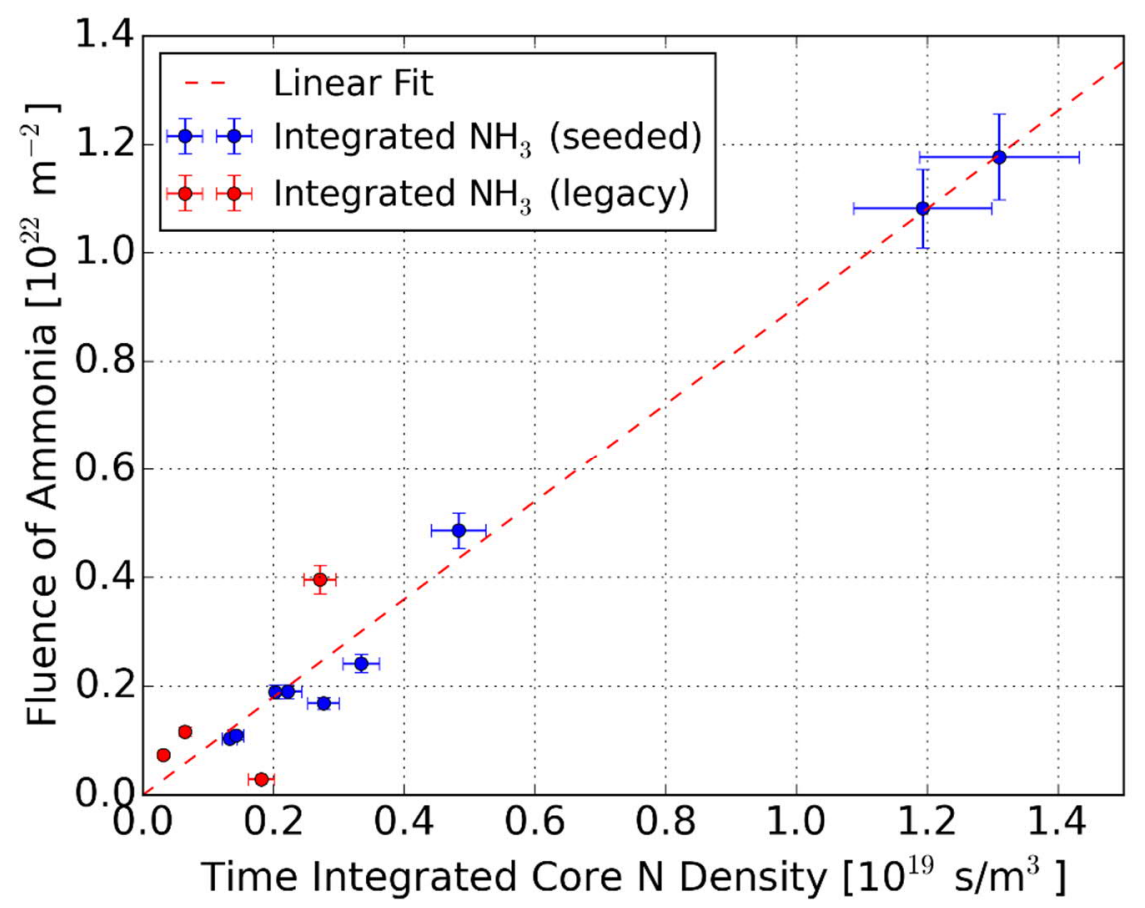

Figure 8. Fluence of ammonia measured with the inner divertor RGA, plotted against pulseintegrated core $\mathrm{N}$ density, for seeded and legacy discharges

\subsection{Model of ammonia formation and correlation to nitrogen content}

A schematic of the $\mathrm{N}_{2}$ and ammonia formation in the neutral gas in $\mathrm{N}_{2}$-seeded and legacy discharges is shown in Figure 9.

As $\mathrm{N}_{2}$ is puffed into the divertor of the fusion device, a part of it enters the plasma, where it is dissociated, and contributes to the nitrogen population in the core and divertor plasma and takes part in the recycling and surface recombination processes. The nitrogen atoms exiting the divertor plasma will, on their way to the RGA, recombine into nitrogen-containing molecules, i.e. $\mathrm{N}_{2}$ and ammonia. As shown in the previous section, another part of the puffed $\mathrm{N}_{2}$ bypasses the plasma and goes straight into the RGA. Thus, it contributes to the molecular $\mathrm{N}_{2}$ signal and cannot be distinguished from the $\mathrm{N}_{2}$ formed from the $\mathrm{N}$ atoms exiting the plasma. Ammonia, on 
the other hand, can only be formed from the $\mathrm{N}$ exiting the plasma, and thus the measured fluence of ammonia can be directly related to the fluence of $\mathrm{N}$ atoms from the divertor plasma.
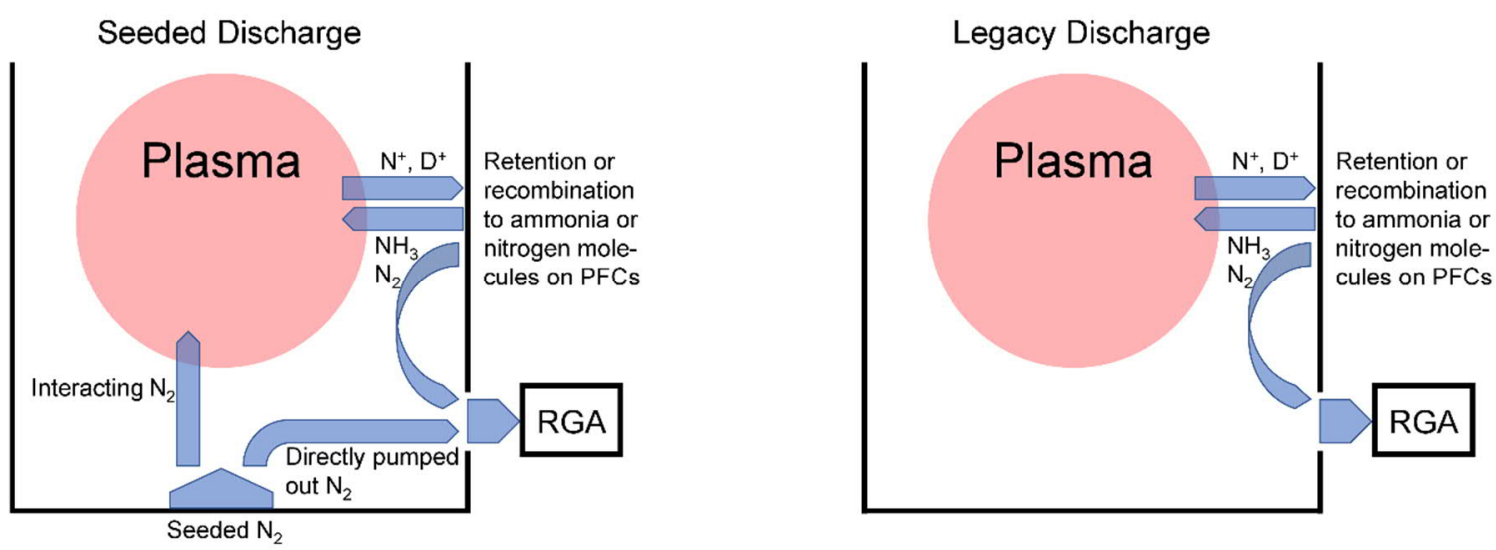

Figure 9. Comparison of nitrogen in seeded and legacy discharge and the formation of ammonia

In a legacy discharge, no $\mathrm{N}_{2}$ is seeded and the $\mathrm{N}$ recovered from the wall inventory is the only source of $\mathrm{N}$ in the plasma. In this case, all $\mathrm{N}$-containing molecules detected by the RGA ( $\mathrm{N}_{2}$ and ammonia) are formed from the $\mathrm{N}$ from the plasma which allows for a direct measurement of the $\mathrm{N}$ fluence as described in equation 4 . Moreover, the legacy discharges allow for the determination of the fraction of the nitrogen atoms which form ammonia molecules, $\delta$ :

$$
\text { Conversion Fraction } \delta=\frac{\phi_{\mathrm{NH}_{3}}}{2 \cdot \phi_{\mathrm{N}_{2}}+\phi_{\mathrm{NH}_{3}}}
$$

Table 4 summarizes the plasma density, plasma current and total heating power of all evaluated legacy discharges. Different $\mathrm{H}$-mode discharges in the lower single null configuration with varying total heating (between 2.5 and $9.3 \mathrm{MW}$ ), plasma density (between 2.9 and $9.0 \cdot 10^{19} \mathrm{~m}^{-3}$ ) and plasma current (between 0.6 and $1.0 \mathrm{MA}$ ) were evaluated. The effect of the magnetic configuration was not studied. Changes in the magnetic configuration result in different distances between the strike points and the inlet ports of the RGAs which would in a limited extent be reflected on the pressures of the detected impurities. However we do not expect that this would affect the conversion fraction beyond the measured uncertainty. Even though basic plasma parameters are varying by a factor of 3 to 4 , the conversion fractions for the evaluated legacy discharges stay constant around $0.49 \pm 0.03$ for the inner divertor and $0.21 \pm 0.03$ for the outer divertor. While this is a significantly higher figure than the $8 \%$ conversion fraction for the inner divertor reported by Neuwirth et al [2], it must be noted that Neuwirth et al. compared the amount of detected ammonia to the total amount of injected $\mathrm{N}_{2}$, and not the fluence of $\mathrm{N}$ atoms from the plasma. The significantly lower conversion fraction reported by Neuwirth et al. is therefore another indication of the bypass of the seeded $\mathrm{N}_{2}$. 


\begin{tabular}{|l|c|c|c|c|c|}
\hline & $\mathbf{3 4 1 6 2}$ & $\mathbf{3 4 1 6 3}$ & $\mathbf{3 4 2 6 9}$ & $\mathbf{3 4 2 7 0}$ & $\mathbf{3 4 5 3 5}$ \\
\hline $\boldsymbol{n}_{\boldsymbol{e}}\left[\cdot \mathbf{1 0}^{\mathbf{1 9}} \mathbf{m}^{-3}\right]$ & 2.86 & - & 6.01 & 7.74 & 8.96 \\
\hline $\boldsymbol{I}_{\boldsymbol{p}}[\mathrm{MA}]$ & 0.80 & 1.00 & 0.60 & 0.80 & 1.02 \\
\hline $\boldsymbol{P}_{\text {tot }}[\mathrm{MW}]$ & 2.49 & 4.95 & 9.26 & 4.89 & 5.08 \\
\hline \hline $\begin{array}{l}\text { Conversion Fraction } \\
\text { inner div. }\end{array}$ & $0.502 \pm 0.022$ & $0.434 \pm 0.021$ & $0.538 \pm 0.022$ & $0.479 \pm 0.022$ & $0.492 \pm 0.022$ \\
\hline $\begin{array}{l}\text { Conversion Fraction } \\
\text { outer div. }\end{array}$ & $0.189 \pm 0.022$ & $0.214 \pm 0.024$ & $0.227 \pm 0.025$ & $0.247 \pm 0.027$ & $0.162 \pm 0.021$ \\
\hline
\end{tabular}

Table 4. Basic plasma parameters and the conversion fractions of the inner and outer divertor measurements for legacy discharges

Table 4 also outlines the difference of the conversion fraction for legacy discharges between inner and outer divertor with a factor of about 2 - 3. A possible explanation is the in-vessel cryo pump which affects the transport of the residual gas to the outer divertor RGA. The cryo pump is composed of a panel cooled to liquid He temperature ( $\mathrm{LHe}$ ), encircled with a shield at the liquid $\mathrm{N}_{2}$ temperature. The aim of the shield is to reduce the pressure of impurities (chiefly water) which would otherwise stick to the LHe panel and thus reduce its efficiency. Because ammonia's vapour pressure is much higher compared to nitrogen, ammonia is frozen onto the $\mathrm{LN}_{2}$ shield, while nitrogen is only absorbed by the LHe panels of the cryo pump. The different pumping efficiency between ammonia and nitrogen of the cryo pump disturbs the comparison of both signals in the conversion fraction.

\subsection{Reconstruction of nitrogen fluence from the divertor plasma during seeded discharges}

The conversion fraction, determined in the legacy discharges, allows for the assessment of the total nitrogen fluence in $\mathrm{N}_{2}$-seeded discharges, as well. Due to the disturbance of the outer divertor measurements the reconstruction calculation of the nitrogen fluence from the divertor plasma is only done for the inner divertor case.

By the application of the mean conversion fraction of legacy discharges onto the measured amount of ammonia, a reconstructed total nitrogen fluence from the divertor plasma can be retrieved, which is the sum of the molecular nitrogen fluence and the ammonia fluence. With help of equation (5) the reconstructed nitrogen fluence from the divertor plasma, $\phi_{N R}$, can be described as:

$$
\phi_{N R}=\frac{\phi_{N_{3}}}{\delta} .
$$


Figure 10 shows that the reconstructed total nitrogen fluence overlaps very well with the measurements during legacy discharges while a large gap is visible during seeded discharge. This could be explained by the bypass (up to $90 \%$ ) of the seeded nitrogen which does not contribute to the ammonia production but is measured in the residual gas. Similar bypass effects were already seen in other studies with ${ }^{13} \mathrm{CH}_{4}$ in JET, where up to $85 \%$ of the gas which was puffed in was lost [6].

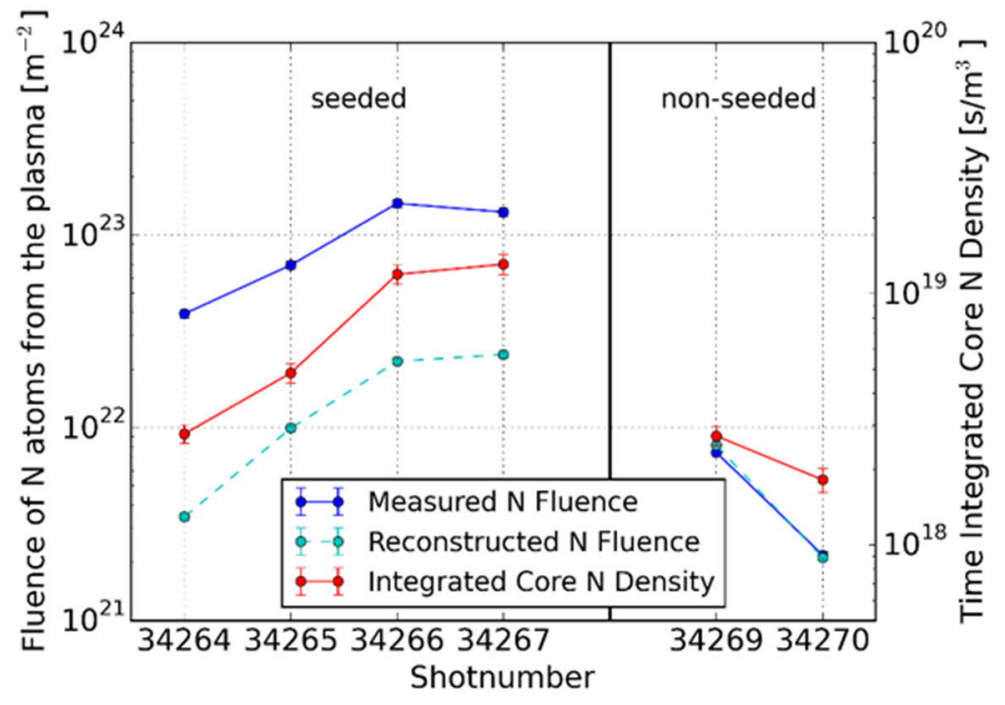

Figure 10. Comparison of measured and reconstructed nitrogen fluence in the inner divertor for the set of discharges $34264-34270$

\section{Summary}

The nitrogen content in the residual gas of seeded and legacy discharges at ASDEX Upgrade has been investigated by mass spectrometry. Absolute and cracking pattern calibration of the devices were done for a quantitative evaluation of the residual gas. For the deconvolution of the residual gases a minimization method was used. Ammonia formation is clearly visible for seeded but also for subsequent non-seeded discharges.

Experimental data shows that the molecular nitrogen $\left(\mathrm{N}_{2}\right)$ fluence measured by the RGAs during seeded discharges is distorted by local effects and bypasses. Therefore, the $\mathrm{N}_{2}$ measurement cannot be used as a direct measurement of the nitrogen atom fluence from the divertor. The fluence of ammonia, on the other hand, is not affected by the local effects, and is generally proportional to the nitrogen density in the core plasma, evaluated by CXRS. Therefore, unlike $\mathrm{N}_{2}$, the fluence of ammonia is a good indicator for the fluence of nitrogen from the plasma.

The measured ammonia and nitrogen fluences during legacy discharges were used to calculate the fraction of the nitrogen atoms which form ammonia. This fraction allows to reconstruct total fluence of nitrogen atoms from the divertor plasma during seeded discharges. Results of the inner divertor data show that in $\mathrm{H}$-mode discharges the nitrogen-to-ammonia conversion fraction can be up to $50 \%$. This seems to be independent of basic plasma parameters as the density and the heating power. However, for a better understanding of dependencies of the conversion 
fraction, the database needs to be extended and more different discharges need to be evaluated.

In the outer divertor a significantly smaller conversion fraction was observed, which is likely due to the local influence of the cryo pump on the residual gas transport to the location of the RGA. This issue has been since addressed with a hardware update of the measurement setup and will provide data for future studies. The reconstructed nitrogen fluences for seeded discharges are a strong indication that a significant fraction of the seeded nitrogen uses bypasses and therefore does not interact with the divertor plasma.

[1] Beurskens MNA, Dunne MG, Frassinetti L, Bernert M, Cavedon M, Fischer R, et al. The role of carbon and nitrogen on the $\mathrm{H}$-mode confinement in ASDEX Upgrade with metal wall. Nucl Fusion. 2016;56(5):056014

[2] Neuwirth D, Rohde V, Schwarz-Selinger T, ASDEX Upgrade team. Formation of ammonia during nitrogen-seeded discharges as ASDEX Upgrade. Plasma Phys. Control. Fusion. 2012;54:085008

[3] Rohde V, Oberkofler M, ASDEX Upgrade team. Ammonia production in nitrogen seeded plasma discharges in ASDEX Upgrade. Journal of Nuclear Materials. 2015;463:672-675

[4] Price GL, Iglesia E. Matrix Method for Correction of Mass Spectra in Deuterium-Exchange Applications. Industrial \& Engineering Chemistry Research. 1989;28(6):839-844

[5] Drenik A, et al. Detection of ammonia by residual gas analysis in AUG and JET. Fusion Engineering and Design. 2017;124:239-243

[6] Likonen J, et al. Deposition of ${ }^{13} \mathrm{C}$ tracer in the JET Mkll-HD divertor. Physica Scripta 2011;T145:014004

[7] Drenik A, et al. Evolution of nitrogen concentration and ammonia production in $\mathrm{N}_{2}$-seeded H-mode discharges at AUG. Nuclear Fusion 2019; 59:04601

[8] Oberkofler $M$, et al. Nitrogen mechanisms in tokamaks with beryllium and tungsten plasma-facing surfaces. Physica Scripta 2016;T167:014077

[9] Viezzer E, et al. High-resolution charge exchange measurements at ASDEX Upgrade. Review of Scientific Instruments 2012;83:103501

[10] Reichbauer T. Assessment of nitrogen enrichment cooled by nitrogen gas puffing. IPP 2018-12. Garching, Max-Planck-Institut für Plasmaphysik. doi:10.17617/2.2596739 (http://hdl.handle.net/21.11116/0000-0001-6A6E-E)

[11] Kallenbach $A$, et al. Divertor power load feedback with nitrogen seeding in ASDEX Upgrade. Plasma Phys. Control. Fusion. 2010;52:055002

[12] Drenik A, et al. Evaluation of the plasma hydrogen isotope content by residual gas analysis at JET and AUG. Physica Scripta 2017;T170:014021 
[13] Drenik A, et al. Mass spectrometry analysis of the impurity content of in N2 seeded discharges in JET-ILW. Journal of Nuclear Materials. 2015;463:684-7

[14] Schweinzer J, et al. Confinement of 'improved H-modes' in all-tungsten ASDEX Upgrade with nitrogen seeding. Nuclear Fusion 2011;55(11):113003

[15] MKS HPQ2 Manual. https://adms.fnal.gov/vacuum/manuals/mksrga/Manuals/Smart\%20Head/LP10 1011.202\%20HPQ-2.pdf

[16] MKS HPQ3 Datasheet. https://www.mksinst.com/mam/celum/celum_assets/resources/HPQ3DS.pdf?1 